\title{
Screening candidate genes associated with bladder cancer using DNA microarray
}

\author{
AXIANG XU, CHUNYANG WANG and SHENGKUN SUN \\ Department of Urology, PLA General Hospital, Beijing 100853, P.R. China
}

Received November 29, 2013; Accepted July 4, 2014

DOI: $10.3892 / \mathrm{mmr} .2014 .2667$

\begin{abstract}
The aim of the present study was to screen candidate genes that are closely associated with bladder cancer and to select the most distinct candidate target genes in order to provide theoretical evidence and direction for improved treatment of bladder cancer. The gene microarray dataset GSE45184 was downloaded from the Gene Expression Omnibus database. There were a total of six expression prolife microarrays from three pairs of freshly frozen bladder cancer tissues and corresponding normal adjacent tissues. Differentially expressed genes (DEGs) were identified using the limma package in $\mathrm{R}$ software and then subjected to further biological information analysis, including hierarchical clustering analysis and gene ontology enrichment analysis. Co-expression networks and functional interaction networks were established using the up- and downregulated genes. Pathway enrichment analysis was then performed for the genes in the functional interaction networks. A total of 522 DEGs were identified, including 223 upregulated and 299 downregulated genes. Functional enrichment analysis of the target genes indicated that downregulated genes were associated with the regulation of biological processes, while the upregulated genes participated in the processes involved in the cell cycle. The functional network of the upregulated genes comprised 1,518 connections and 92 gene nodes that were associated with 10 closely-related functions, while the network of the downregulated genes consisted of 129 connections and 24 gene nodes involving 11 significantly related functions. Pathway enrichment analysis revealed that the downregulated genes were mainly involved in the mitogen-activated protein kinase signaling pathway, while the upregulated genes were closely associated with the cell cycle. These DEGs and the relevant cell cycle pathways have the potential to be used as targets for the treatment of bladder cancer.
\end{abstract}

Correspondence to: Dr Axiang Xu, Department of Urology, PLA General Hospital, 28 Fuxing Road, Beijing 100853, P.R. China E-mail: xuaxiang0958@foxmail.com

Key words: bladder cancer, differentially expressed genes, functional interaction network, gene ontology enrichment analysis, pathway enrichment analysis

\section{Introduction}

Bladder cancer is the second most common genitourinary malignancy, according to a study by Stein et al (1). It was estimated in 1999 that 54,200 diagnoses of bladder cancer were made annually, and that the annual mortality at that time was $\sim 12,100$ (2). Although the majority of patients are initially diagnosed with superficial bladder tumors, a considerable percentage (20-40\%) present with or develop into an invasive form of the disease. Locally advanced or metastatic bladder cancer is not easily treated, and therefore, research into the molecular mechanisms involved in bladder cancer is required in order to provide important insights into how normal cells become tumorigenic, and to promote the development of novel cancer treatment strategies.

Previously, a considerable number of studies have focused on bladder cancer $(3,4)$. They have indicated that various angiogenic factors, including vascular endothelial growth factor, platelet-derived endothelial cell growth factor, acidic-fibroblast growth factor (FGF) and basic-FGF are involved in the tumor angiogenesis and invasion of bladder cancer (1,5-8). Additionally, a study regarding transitional-cell carcinoma and the growth regulation of bladder cancer cells has made notable progress (1). However, the mechanism underlying the occurrence and development of bladder cancer remains unclear. Therefore, further study of the molecular mechanisms underlying bladder cancer is required, which has the potential to contribute to the development of novel treatment strategies.

In the current study, microarrays were utilized to identify differentially expressed genes (DEGs) between cells obtained from bladder cancer and normal tissues. The functional annotations of DEGs were investigated to determine the biological process and pathways. Additionally, the study aimed to determine co-expression and functional networks of the up- and downregulated genes.

\section{Materials and methods}

Microarray data and data preprocessing. The microarray dataset GSE45184 (9) was downloaded from the Gene Expression Omnibus (GEO) database (http://www.ncbi.nlm. nih.gov/geo/), which included six microarray expression profiles from three pairs of freshly frozen bladder cancer tissues and corresponding adjacent normal tissues. The platform GPL14550 Agilent-028004 SurePrint G3 Human GE 8x60K 
Microarray [Probe Name Version, Agilent Technologies, Palo Alto, CA, USA, (http://www.genome.jp/kegg/pathway.html)] was also downloaded from the GEO database. Subsequent to the input of missing data, the probe-level data were converted into expression measures using the annotation information in the GPL14550 platform.

In the situation of one gene corresponding with more than one probe (multiple expression values), the average value was calculated as the only corresponding expression value. The gene expression data were transformed to a normal distribution from a skewed distribution using log base- 2 and were then normalized with the median method (10).

Differential expression analysis. The limma package (Affymetrix, Santa Clara, California http://www.affymetrix. com/analysis/) (11) was used to identify DEGs between the bladder cancer and control samples. Genes with $\mathrm{P} \leq 0.05$ and $\mid \log \mathrm{FCl}>1$ were selected as the DEGs for further analysis. Hierarchical clustering was then performed for all DEGs (12) and the clusters were visualized with TreeView software (http://treeview1.software.informer.com/) (13).

Construction of co-expressed gene networks. COXPRESdb (http://coxpresdb.jp/), is a database of co-expressed gene sets that provides valuable information for a wide variety of experimental procedures, including the targeting of genes for functional identification, gene regulation and protein-protein interactions (14). In the present study, the DEGs were divided into two sets, the upregulated and the downregulated genes. The co-expression networks of these two groups of genes were established based on the information from COXPRESdb. The coexpressed genes that presented a correlation coefficient $>0.7$ were selected for further analysis.

Gene Ontology function enrichment analysis. WebGestalt (http://bioinfo.vanderbilt.edu/webgestalt/) was used for the function enrichment analysis of up- and downregulated genes (15). $\mathrm{P} \leq 0.05$ was considered to indicate a statistically significant difference.

Establishment of functional interaction networks. The information on GO functions that was obtained was integrated into the co-expression networks using COXPRESdb database to form functional interaction networks of the up- and downregulated genes.

Kyoto Encyclopedia of Genes and Genomes (KEGG) pathway analysis for the genes in the functional interaction networks. According to the pathway information from the Database for Annotation, Visualization and Integrated Discovery (DAVID; DAVID Bioinformatics Resources 6.7, http://david.abcc.ncifcrf. gov/home.jsp), all genes constituting a functional network were subjected to pathway analysis based on the KEGG (http://www. genome.jp/kegg/pathway.html) (16). $\mathrm{P} \leq 0.05$ was considered to indicate a statistically significant difference.

\section{Results}

Differential expression analysis. Following preprocessing of data, difference comparison was performed using the normal- ized data. All DEGs with $\mathrm{P} \leq 0.05$ and $\mid \log \mathrm{FCl}>1$ were selected, with the normal samples as the control group. In total, 522 genes, including 299 downregulated and 223 upregulated genes were identified (Table I).

Hierarchical clustering based on the expression measures of the DEGs generated a tree with a clear distinction between the cancer samples and normal samples (Fig. 1).

Establishment of co-expression networks of up- and downregulated genes. The DEGs were split into two sets, one with 299 downregulated genes and the other with 223 upregulated genes. Co-expression networks were constructed among the DEGs in each set with the COXPRESdb database. The co-expression gene network of the upregulated gene set consisted of 4,021 connections and the network of the downregulated genes consisted of 111 connections.

GO enrichment analysis of DEGs. WebGestalt software was applied to perform GO functional enrichment analysis for the DEGs in the two sets. Table I and Fig. 1 indicate that the up- and downregulated genes were mainly enriched in the cell cycle and regulation of system processes, respectively.

Establishment of functional interaction networks. The data of the GO nodes were integrated with that of the co-expression networks in order to construct functional interaction networks for the up- and downregulated genes. The network of upregulated genes comprised 1,518 connections and 92 gene nodes that were associated with 10 closely-related functions, while the downregulation network consisted of 129 connections and 24 gene nodes, which involved 11 significantly related functions (Fig. 2).

Pathway enrichment analysis of genes in the functional network. The 82 upregulated and 36 downregulated genes of the functional networks were input into DAVID to identify the overrepresented KEGG pathways. As presented in Table II, the downregulated genes were mainly associated with four pathways, and the most significantly overrepresented pathway was the mitogen-activated protein kinase (MAPK) signaling pathway. A total of 9 pathways were enriched by the 82 upregulated genes, among which the cell cycle was the most significant, involving 24 genes.

\section{Discussion}

Bladder cancer is one of the most common malignancies of the urinary system. The occurrence and development of bladder cancer are regulated by multiple genetic and biological processes. However, the mechanism of its occurrence and development have not been elucidated by previous studies. The present study selected candidate genes that were closely associated with bladder cancer by comparing the expression profiles of samples from cancer and control tissues. A total of 522 genes, including 299 downregulated and 223 upregulated genes were obtained. Functional networks for the upregulated and downregulated genes were constructed, and pathway enrichment analysis revealed that the up- and downregulated genes were mainly enriched in the cell cycle and the MAPK signaling pathway, respectively. 
Table I. GO enrichment analysis of up- and downregulated genes.

A, Downregulated genes

\begin{tabular}{lll}
\hline GO term ID & \multicolumn{1}{c}{ GO term description } & P-value \\
\hline GO:0044057 & Regulation of system processes & $4.17 \mathrm{E}-06$ \\
GO:0006937 & Regulation of muscle contraction & $2.63 \mathrm{E}-03$ \\
GO:0006940 & Regulation of smooth muscle contraction & $2.71 \mathrm{E}-03$ \\
GO:0007517 & Muscle organ development & $8.56 \mathrm{E}-03$ \\
GO:0003012 & Muscle system process & $1.96-\mathrm{E} 02$ \\
GO:0009725 & Response to hormone stimulus \\
GO:0006936 & Muscle contraction & $2.68 \mathrm{E}-02$ \\
GO:0008201 & Heparin binding & $4.30 \mathrm{E}-02$ \\
GO:0030247 & Polysaccharide binding & $1.24 \mathrm{E}-02$ \\
GO:0001871 & Pattern binding & $1.55 \mathrm{E}-02$ \\
GO:0005539 & Glycosaminoglycan binding & $1.55 \mathrm{E}-02$ \\
& & $3.35 \mathrm{E}-02$ \\
\hline
\end{tabular}

B, Upregulated genes

\begin{tabular}{llr}
\hline GO term ID & \multicolumn{1}{c}{ GO term description } & P-value \\
\hline GO:0007049 & Cell cycle & $9.90 \mathrm{E}-52$ \\
GO:0000279 & M phase & $3.33 \mathrm{E}-47$ \\
GO:0022403 & Cell cycle phase & $4.56 \mathrm{E}-45$ \\
GO:0007067 & Mitosis & $7.28 \mathrm{E}-43$ \\
GO:0000280 & Nuclear division & $7.28 \mathrm{E}-43$ \\
GO:0000087 & M phase of mitotic cell cycle & $1.85 \mathrm{E}-42$ \\
GO:0048285 & Organelle fission & $5.79 \mathrm{E}-42$ \\
GO:0022402 & Cell cycle process & $1.80 \mathrm{E}-41$ \\
GO:0000278 & Mitotic cell cycle & $3.01 \mathrm{E}-40$ \\
GO:0051301 & Cell division & $4.02 \mathrm{E}-35$ \\
\hline
\end{tabular}

GO, gene ontology.

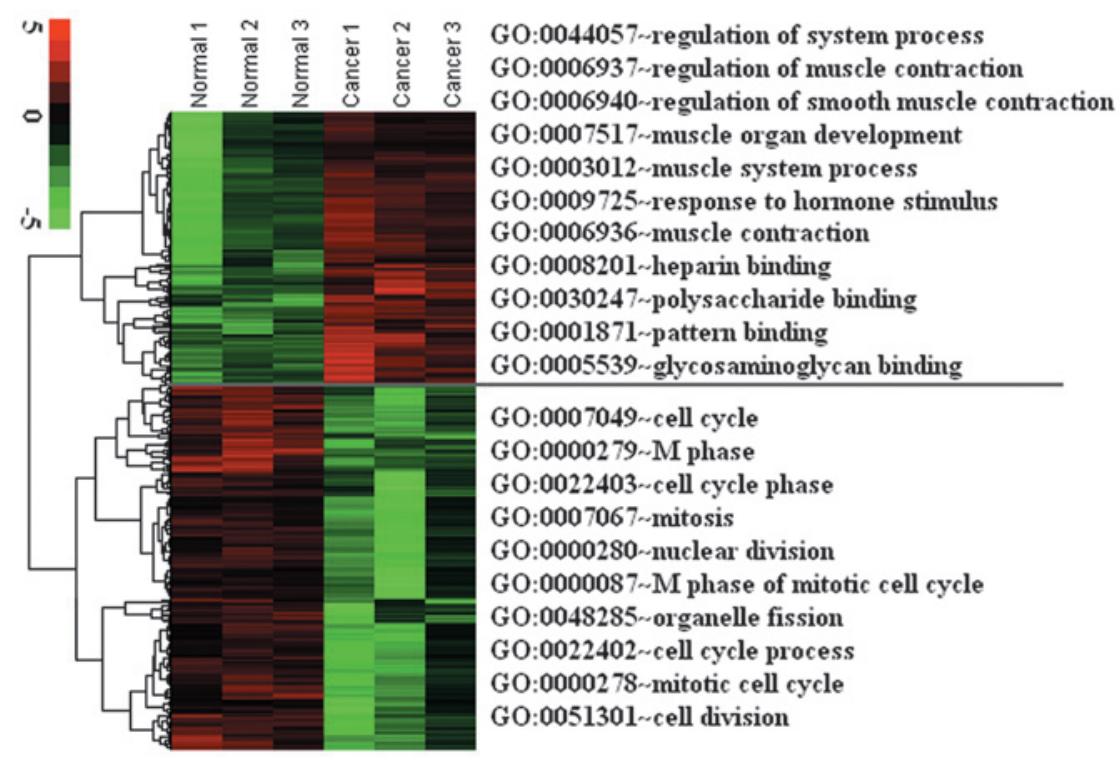

Figure 1. Clustering and GO enrichment analyses of the DEGs. Left: Clustering analysis. The red color represents genes with high expression values, while green represents low expression values. The color change from green to red indicated the change of expression values from low to high. The horizontal axis represents DEGs and the vertical axis represents the different samples. Right: GO enrichment analysis. The top half includes GO functions enriched by upregulated genes and the bottom half indicates those enriched by downregulated genes. GO, gene ontology; DEG, differentially expressed gene. 
Table II. Kyoto Encyclopedia of Genes and Genomes pathway enrichment analysis of the genes in the functional interaction networks.

A, Downregulated genes

\begin{tabular}{llr}
\hline Term & Count & P-value \\
\hline hsa04010:MAPK signaling pathway & 9 & $5.22 \mathrm{E}-03$ \\
hsa05414:Dilated cardiomyopathy & 5 & $1.40 \mathrm{E}-02$ \\
hsa04610:Complement and coagulation cascades & 4 & $3.29 \mathrm{E}-02$ \\
hsa05020:Prion diseases & 3 & $4.92 \mathrm{E}-02$ \\
\hline
\end{tabular}

B, Upregulated genes

\begin{tabular}{lcr}
\hline Term & Count & P-value \\
\hline hsa04110:Cell cycle & 24 & $2.18 \mathrm{E}-25$ \\
hsa04114:Oocyte meiosis & 14 & $1.14 \mathrm{E}-11$ \\
hsa04914:Progesterone-mediated oocyte maturation & 10 & $7.25 \mathrm{E}-08$ \\
hsa04115:p53 signaling pathway & 8 & $2.66 \mathrm{E}-06$ \\
hsa03030:DNA replication & 5 & $3.30 \mathrm{E}-04$ \\
hsa03410:Base excision repair & 4 & $4.18 \mathrm{E}-03$ \\
hsa00240:Pyrimidine metabolism & 5 & $1.19 \mathrm{E}-02$ \\
hsa03440:Homologous recombination & $5.82 \mathrm{E}-02$ \\
hsa00790:Folate biosynthesis & 3 & $9.92 \mathrm{E}-02$ \\
\hline
\end{tabular}
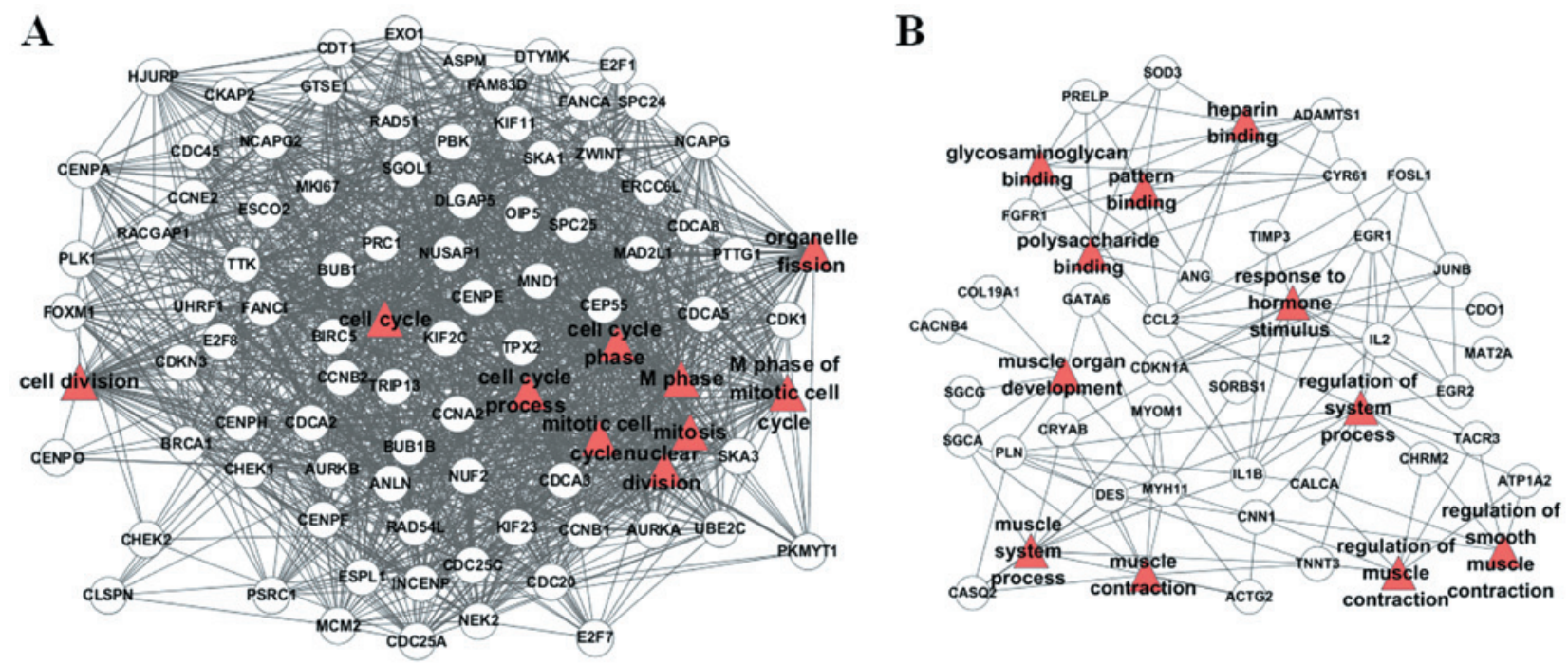

Figure 2. Functional interaction networks constructed for the (A) upregulated and (B) downregulated genes. Triangle nodes represent the common function of related genes and the cycle nodes represent genes.

Regardless of the complexity and individuality of each type of cancer, there are a limited number of critical events that promote the tumor cell and its progeny into uncontrolled expansion and invasion (17). One of these is the deregulated cell cycle. Cell cycle progression is a highly ordered and tightly-regulated process that involves multiple checkpoints that assess extracellular growth signals, cell size and DNA integrity (18). In normal tissues, cells have established a series of systems to ensure the normal functioning of the cell cycle. However, when a cell becomes a neoplasm, its normal cell cycle is disturbed, alongside the suppression of growth and apoptosis required to support it (19). As such, it is not surprising that deregulation of the cell cycle in bladder cancer is one of the most frequent abnormal occurrences during its development (20). Therefore, skillful targeting of these critical events may lead to the development of potent and specific therapeutic strategies for use in bladder cancer.

The cell cycle of eukaryotic cells is regulated by a family of protein kinases termed cyclin-dependent kinases (CDKs) $(21,22)$. Cell cycle progression is determined by the proteins that are 
activated or inactivated, which is a result of the activity of the CDKs during that stage. In normal cells, a succession of kinase subunits (CDK4, CDK6, CDK2 and CDC2) are expressed along with a succession of cyclins (cyclin D, E, A and B). Therefore, CDKs and their cyclin partners are positive regulators or accelerators that induce cell cycle progression (20). A previous study indicated that CDK1 may have an important role in a diverse range of biological processes, including cancer-cell invasion and osteogenic differentiation of mesenchymal stem cells (23). In the current study, GO function and KEGG pathway enrichment analysis demonstrated that CDK1 was involved in the cell cycle and may be the critical target gene required for bladder cancer, providing novel approaches for the treatment of bladder cancer.

Furthermore, MAPK cascades associated with the functions of downregulated genes are crucial in cancer as they influence the cell regulation system. Cyclin D regulates $\mathrm{G}_{1}$ phase progression in cancer cells and is overexpressed in various malignant neoplasms (24). As a result, it is a potential target for cancer therapeutics (18). Transcriptional regulation of cyclin D has been extensively investigated and is well understood (25-27). It is stimulated when various mitogenic signals activate the Ras/Raf/MEK/ERK (MAPK) cascade. Following synthesis, subsequent to MAPK cascade activation, cyclin D acts with CDK4 and CDK6 in the cyclin D-CDK4/CDK6 pathway, to regulate the cell through the critical point of the $\mathrm{G}_{1}$ stage $(28,29)$. Therefore, the Ras-MAPK pathway is important for orchestrating cell responses to external and internal stimuli. This pathway is commonly deregulated in cases of cancer, including those of bladder cancer (30). Numerous drugs that target the Ras-MAPK pathway are being investigated in phase II clinical trials for bladder cancer, alone or in combination with other chemotherapeutic agents (31).

The up- and downregulated genes were closely associated with cell cycle functions in bladder cancer. Therefore, understanding the molecular mechanisms underlying the deregulation of cell cycle progression in bladder cancer may provide important insight into how normal cells become tumorigenic, in addition to how treatment strategies can be designed to target bladder cancer.

\section{References}

1. Stein JP, Lieskovsky G, Cote R, et al: Radical cystectomy in the treatment of invasive bladder cancer: long-term results in 1,054 patients. J Clin Oncol 19: 666-675, 2001.

2. Tsihlias $J$ and Grossman HB: The utility of fibrin/fibrinogen degradation products in superficial bladder cancer. Urol Clin North Am 27: 39-46, 2000.

3. Sutcliffe S and Colditz GA: Prostate cancer: is it time to expand the research focus to early-life exposures? Nat Rev Cancer 13: 208-518, 2013.

4. Troyer D: Emerging biomarkers in prostate cancer. Aging Health 2: 579-588, 2006.

5. O'Brien T, Cranston D, Fuggle S, Bicknell R and Harris AL: Different angiogenic pathways characterize superficial and invasive bladder cancer. Cancer Res 55: 510-513, 1995.

6. Nguyen M, Watanabe H, Budson AE, Richie JP and Folkman J: Elevated levels of the angiogenic peptide basic fibroblast growth factor in urine of bladder cancer patients. J Natl Cancer Inst 85: $241-242,1993$
7. Chopin DK, Caruelle JP, Colombel M, et al: Increased immunodetection of acidic fibroblast growth factor in bladder cancer, detectable in urine. J Urol 150: 1126-1130, 1993.

8. Campbell SC: Advances in angiogenesis research: relevance to urological oncology. J Urol 158: 1663-1674, 1997.

9. He W, Cai Q, Sun F, et al: linc-UBC1 physically associates with polycomb repressive complex 2 (PRC2) and acts as a negative prognostic factor for lymph node metastasis and survival in bladder cancer. Biochim Biophys Acta 1832: 1528-1537, 2013.

10. Fujita A, Sato JR, Rodrigues L de O, Ferreira CE and Sogayar MC: Evaluating different methods of microarray data normalization. BMC Bioinformatics 7: 469, 2006.

11. Gentleman R, Carey VJ, Huber W, Irizarry RA, and Dudoit S (eds): Bioinformatics and Computational Biology Solutions Using R and Bioconductor. Vol 746718470. Springer, New York, NY, 2005 XIX.

12. Eisen MB, Spellman PT, Brown PO and Botstein D: Cluster analysis and display of genome-wide expression patterns. Proc Natl Acad Sci USA 95: 14863-14868, 1998.

13. Saldanha AJ: Java Treeview - extensible visualization of microarray data. Bioinformatics 20: 3246-3248, 2004.

14. Obayashi T, Hayashi S, Shibaoka M, Saeki M, Ohta H and Kinoshita K: COXPRESdb: a database of coexpressed gene networks in mammals. Nucleic Acids Res 36: D77-D82, 2008.

15. Sherman BT and Lempicki RA: Bioinformatics enrichment tools: paths toward the comprehensive functional analysis of large gene lists. Nucleic Acids Res 37: 1-13, 2009.

16. Huang da W, Sherman BT and Lempicki RA: Systematic and integrative analysis of large gene lists using DAVID bioinformatics resources. Nat Protoc 4: 44-57, 2009.

17. Evan GI and Vousden KH: Proliferation, cell cycle and apoptosis in cancer. Nature 411: 342-348, 2001.

18. Ruzinova MB and Benezra R: Id proteins in development, cell cycle and cancer. Trends Cell Biol 13: 410-418, 2003.

19. Hartwell LH and Kastan MB: Cell cycle control and cancer. Science 266: 1821-1828, 1994.

20. Park MT and Lee SJ: Cell cycle and cancer. J Biochem Mol Biol 36: 60-65, 2003.

21. Serrano M, Hannon GJ and Beach D: A new regulatory motif in cell-cycle control causing specific inhibition of cyclin D/CDK4. Nature 366: 704-707, 1993.

22. Takuwa N and Takuwa Y: Regulation of cell cycle molecules by the Ras effector system. Mol Cell Endocrinol 177: 25-33, 2001.

23. Wei Y, Chen YH, Li LY, et al: CDK1-dependent phosphorylation of EZH2 suppresses methylation of $\mathrm{H} 3 \mathrm{~K} 27$ and promotes osteogenic differentiation of human mesenchymal stem cells. Nat Cell Biol 13: 87-94, 2011.

24. Malumbres M and Barbacid M: To cycle or not to cycle: a critical decision in cancer. Nat Rev Cancer 1: 222-231, 2001.

25. Tetsu $\mathrm{O}$ and McCormick F: Beta-catenin regulates expression of cyclin D1 in colon carcinoma cells. Nature 398: 422-426, 1999.

26. Tetsu $\mathrm{O}$ and McCormick F: Proliferation of cancer cells despite CDK2 inhibition. Cancer cell 3: 233-245, 2003.

27. Sherr CJ and Roberts JM: Living with or without cyclins and cyclin-dependent kinases. Genes Dev 18: 2699-2711, 2004.

28. LaBaer J, Garrett MD, Stevenson LF, et al: New functional activities for the p21 family of CDK inhibitors. Genes Dev 11: 847-862, 1997.

29. Cheng M, Olivier P, Diehl JA, et al: The p21(Cip1) and p27(Kip1) CDK 'inhibitors' are essential activators of cyclin D-dependent kinases in murine fibroblasts. EMBO J 18: 1571-1583, 1999.

30. Dunn KL, Espino PS, Drobic B, et al: The Ras-MAPK signal transduction pathway, cancer and chromatin remodeling. Biochem Cell Biol 83: 1-14, 2005.

31. Dangle PP, Zaharieva B, Jia H and Pohar KS: Ras-MAPK pathway as a therapeutic target in cancer - emphasis on bladder cancer. Recent Pat Anticancer Drug Discov 4: 125-136, 2009. 